\title{
Role of Impaired Oxalate Homeostasis in Cardiovascular Disease in Patients With End-Stage Renal Disease: An Opinion Article
}

\author{
Natalia Stepanova* \\ State Institution "Institute of Nephrology National Academy of Medical Science of Ukraine", Kyiv, Ukraine
}

Keywords: oxalate homeostasis, cardiovascular disease, end-stage renal disease, oxidative stress, inflammation

\section{INTRODUCTION}

Oxalate is an ionic form of a potentially toxic oxalic acid that is formed in the human body from a combination of food sources and their absorption in the gastrointestinal tract (approximately 30\%), as well as the endogenous synthesis of glyoxylate (40\%) and ascorbic acid (30\%) (Jonassen et al., 2005; Robijn et al., 2011). The balance of oxalate in the human body is achieved due to its renal (up to 90\%) and intestinal (10\%) excretion (Robijn et al., 2011; Huang et al., 2020). Loss of kidney function decreases renal oxalate clearance and increases plasma oxalic acid (POx) concentration, according to

OPEN ACCESS

Edited by: Norberto Perico, Istituto di Ricerche Farmacologiche Mario Negri (IRCCS), Italy

Reviewed by:

Kristina Penniston, University of Wisconsin-Madison,

United States

${ }^{*}$ Correspondence:

Natalia Stepanova nmstep88@gmail.com

Specialty section:

This article was submitted to

Renal Pharmacology,

a section of the journal

Frontiers in Pharmacology

Received: 09 April 2021

Accepted: 17 May 2021

Published: 28 May 2021

Citation:

Stepanova N (2021) Role of Impaired

Oxalate Homeostasis in

Cardiovascular Disease in Patients

With End-Stage Renal Disease: An

Opinion Article.

Front. Pharmacol. 12:692429.

doi: 10.3389/fphar.2021.692429 the progression of chronic kidney disease (CKD) stages (Robijn et al., 2011; Perinpam et al., 2017). The accumulation of oxalate may be associated with oxidative stress, inflammation (Khan, 2014; Ermer et al., 2016; Dominguez-Gutierrez et al., 2018; Korol et al., 2021) and a high risk of cardiovascular disease (CVD) (Liu et al., 2014; Fan X et al., 2017; Arafa et al., 2020; Demikhov et al., 2020) in patients with kidney stones. However, although impaired oxalate homeostasis is a well-known occurrence in patients with end-stage renal disease (ESRD), a high POx concentration has never been considered a trigger for oxidative stress, systemic inflammation, and CVD risk in these patients. In this opinion article, based on the published data and results of our clinical studies, we outlined the possible contribution of oxalate to oxidative stress, chronic inflammation, and CVD risk in patients with ESRD.

\section{Gut Microbiota Disruption Causes Hyperoxalemia in Patients With ESRD}

Given that oxalate is mainly excreted by the kidneys, dialysis seems to be the main approach for oxalate removal in patients with ESRD (Ermer et al., 2017; Perinpam et al., 2017). Nevertheless, despite a homogeneous patient population and a standardized dialysis regimen, the intraindividual predialysis POx concentration level varies from $1.8 \mathrm{mg} / \mathrm{L}(20 \mu \mathrm{mol} / \mathrm{L})$ to $5.4 \mathrm{mg} / \mathrm{L}(60 \mu \mathrm{mol} / \mathrm{L})$ in different studies (Ermer et al., 2017; Perinpam et al., 2017; Korol et al., 2021). Considering the marginal dependence of oxalate homeostasis on its dietary intake (Mitchell et al., 2019; Kumar et al., 2021) and limited renal excretion in patients with ESRD, it remains unclear why they have significant differences in POx concentration under the same treatment conditions. We hypothesized that in patients with anuria/kidney failure, the gut plays a much more significant role in oxalate handling than in healthy participants. Both paracellular and transcellular intestinal oxalate transport

Abbreviations: CKD, chronic kidney disease; CVD, cardiovascular disease; GFR, glomerular filtration rate; IS, indoxyl sulfate; ODA, oxalate-degrading activity. 
disruption and less functional activity of oxalate-degrading bacteria $(\mathrm{ODB})$ in fecal microbiota might be the main factors affecting oxalate homeostasis in patients on dialysis.

The gastrointestinal tract plays a complex role in oxalate metabolism via intestinal oxalate transport and the ability of ODB to degrade oxalate (Hatch and Freel, 2008; Robijn et al., 2011; Huang et al., 2020; Ticinesi et al., 2020). Oxalate is absorbed from all parts of the gastrointestinal tract through paracellular (predominantly passive) and active transcellular mechanisms (Robijn et al., 2011; Ticinesi et al., 2020). The relative contribution of these two transport mechanisms varies with the intestinal segment and its condition (Hatch and Freel, 2008; Robijn et al., 2011; Whittamore and Hatch, 2017). Paracellular transport depends on the residence time of the chyme in the small intestine and the degree of calcium ionization (Hatch and Freel, 2008; Robijn et al., 2011; Whittamore and Hatch, 2017). The active transcellular oxalate flux is derived from anion exchange proteins belonging to the multifunctional SLC26 gene family. One of the gene family members, Slc26a6, is expressed at high levels in the intestine and proximal renal tubules and plays a major role in controlling systemic oxalate metabolism (Whittamore and Hatch, 2017). Oxalobacter formigenes produce a small protein that directly induces oxalate transport via the oxalate transporter SLC26A6-dependent mechanism in intestinal Caco-2 cells (Arvans et al., 2017).

Among oxalate transporters, many factors are involved in determining oxalate absorption and secretion in the gut in patients with ESRD: 1) dietary restriction; 2) high uremic toxin concentration; 3) malabsorption; 4) low blood concentrations of calcium, magnesium, and fiber that may affect the oxalate absorption; 5) use of antibiotics, phosphate binders, or other medications that can influence the quantitative and qualitative composition of gut microbiota; and 6) oxalatedegrading activity (ODA) of gut microbiota (Robijn et al., 2011; Mitchell et al., 2019; Ticinesi et al., 2020).

Gut microbiota is a critical factor affecting intestinal oxalate metabolism and kidney stone formation (Stepanova et al., 2018; Chen et al., 2019; Ticinesi et al., 2020). O. formigenes degrade oxalate in the intestine and stimulate its endogenous secretion (Hatch et al., 2006; Arvans et al., 2017). However, the absence of intestinal $O$. formigenes colonization may not be the only cause for kidney stones, and a diversity of gut ODB (e.g., Lactobacillus spp., Bifidobacterium spp., Bacillus spp., E. faecalis, N. albigula) were identified (Gomathi et al., 2014; Barnett et al., 2016; Miller et al., 2019; Stepanova et al., 2021). Since the gut microbiota in patients on dialysis is characterized by increased Enterobacteriaceae and low colonization of Bifidobacterium and Lactobacillus species compared with normal controls ( $\mathrm{Hu}$ et al., 2020; Ren et al., 2020), the low abundance of ODB in gut microbiota may play a significant role in oxalate homeostasis in patients with ESRD. Indeed, we previously demonstrated that the ODB number in patients on dialysis was significantly lower than in healthy volunteers (Stepanova et al., 2020a; Stepanova et al., 2021). However, when we separately evaluated the ODB number and their total ODA in fecal microbiota in patients on dialysis, we got surprising results. The ODB number was associated neither with their total ODA in fecal microbiota nor urinary oxalate (UOx) excretion and POx concentration. According to the results, only total fecal ODA was associated with urine and plasma oxalate levels: the lower the ODA in the fecal microbiota, the higher the POx concentration and the lower the UOx excretion. Thus, total ODA in the fecal microbiota rather than the ODB number resulted in elevation of plasma and urine oxalate concentrations in patients with ESRD.

\section{Crosstalk Between Oxalate Homeostasis, Oxidative Stress, Inflammation, and CVD Risk in Patients With ESRD}

Patients with kidney stone disease have an increased CVD risk, probably due to the common origin of both diseases (Liu et al., 2014; Fan et al., 2017; Arafa et al., 2020). This common source is likely the gut microbiota (Cheungpasitporn et al., 2014; Ma and Li, 2018; Arafa et al., 2020; Zhou et al., 2020). Many mechanisms have been suggested to be involved in gut microbiota-mediated lipid metabolism disruption, including Bifidobacteria- and Lactobacillus-mediated fermentation of nondigestible carbohydrates, trimethylamine $\mathrm{N}$-oxide-mediated impact of lipid absorption and cholesterol homeostasis, reduction of the total bile acids, oxidative stress initiation, immune cell recruitment, and differentiation ( $\mathrm{Ma}$ and Li, 2018; MateyHernandez et al., 2018; Wolf and Ley, 2019; Zhou et al., 2020). Extrapolating the presented hypothesis to the relationship between impaired oxalate homeostasis and CVD in patients on dialysis, we believe that gut dysbiosis may lead to intestinal barrier dysfunction, disruption of oxalate transport mechanisms in the intestinal epithelium (both secretion and absorption), initiation of oxidative processes, chronic inflammation, and dyslipidemia. In line with the common origin hypothesis, our pilot study findings provide preliminary evidence that a decrease in total ODA in the fecal microbiota is associated with atherogenic dyslipidemia in patients with ESRD (Stepanova et al., 2020b). A proposed mechanism for the interaction between oxalate homeostasis and CVD in patients with ESRD is illustrated in Figure 1.

Oxidative stress and inflammation are major risk factors for accelerated atherosclerosis (Khan, 2014; Yang et al., 2017; Devarajan, 2018). However, excessive renal accumulation of $\mathrm{CaOx}$ crystals may also lead to the formation of reactive oxygen species (ROS) and the activation of oxidative processes and oxidative stress (Khan, 2004, 2014; Abhishek et al., 2017). ROS produced by activated NADPH oxidase due to oxalate dyshomeostasis oxidizes low-density lipoprotein (LDL) to minimally modified LDL (mm-LDL), which induces the secretion of various cytokines, including monocyte chemoattractant protein-1 (MCP-1) (Khan, 2004; Khan, 2014; Abhishek et al., 2017). MCP-1 recruits monocytes and transforms them into foam cells, owing to the modified lipoprotein (Khan, 2004; Khan, 2014; Abhishek et al., 2017). In addition to MCP-1, oxidatively modified LDL induces the synthesis of other proinflammatory cytokines, such as interleukin-6 (IL-6) and tumor necrosis factor-alpha (TNF- $\alpha$ ) (Abhishek et al., 2017; Wolf and Ley, 2019). However, these mediators are strongly 


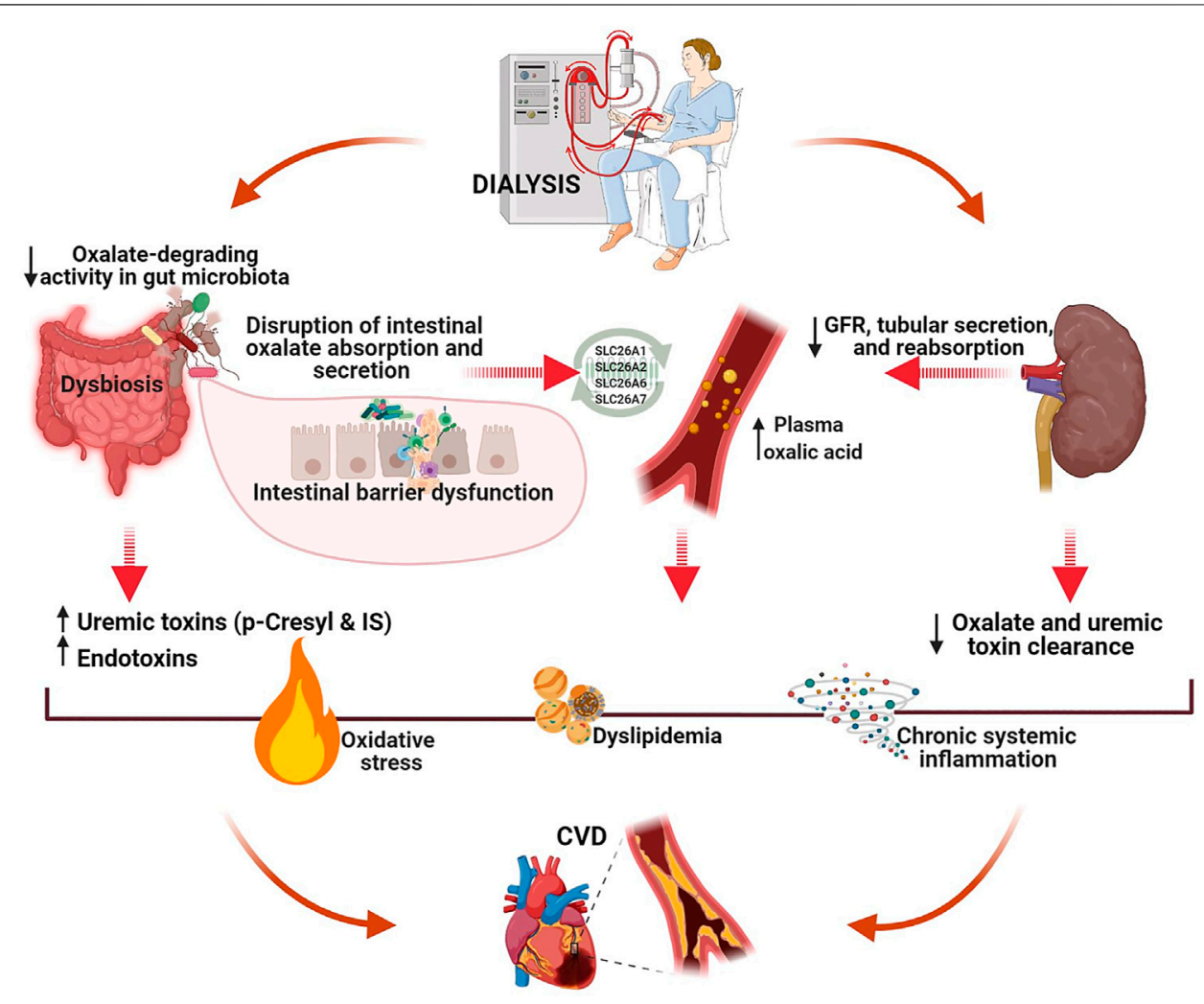

FIGURE 1 | A proposed mechanism of the interaction between oxalate homeostasis and CVD in patients with end-stage renal disease (created with BioRender. com). Intestine plays a crucial role in oxalate homeostasis disruption in patients on dialysis. CKD-associated gut dysbiosis with commensal microbiota deficiency leads to decreased total fecal ODA, intestinal barrier dysfunction, and alteration of oxalate transport mechanisms in the intestinal epithelium (both secretion and absorption). Both uremia- and oxalate-induced molecular mechanisms initiated in the gut can activate oxidative stress, chronic inflammation, and atherogenesis, eventually resulting in CVD.

involved in oxalate-induced inflammation (Găseniuk et al., 2013; Khan, 2014; Abhishek et al., 2017). CaOx can activate human monocytes, which increase TNF- $\alpha$, IL-6, and MCP-1 production and lead to chronic inflammation (Dominguez-Gutierrez et al., 2018). In mirror to mentioned above, our recent study was the first to demonstrate the increasing trend in serum malondialdehyde and oxidative stress index according to the gradual increase in POx concentrations in patients with ESRD (Korol et al., 2021). Moreover, blood antioxidant balance has been associated with the POx concentration: the higher the POx concentration, the more pronounced the decreased levels of ceruloplasmin, $\mathrm{SH}$-groups, and total peroxidase activity. In another study, we separately characterized the association of POx concentration with the lipid profile and proinflammatory markers in patients with ESRD, and identical results were obtained (Stepanova et al., 2020c). POx elevation was significantly associated with an increasing trend in the atherogenic lipoprotein fractions and proinflammatory markers and linearly decreased high-density lipoproteins. Moreover, POx concentration was directly correlated with blood triglycerides, IL-6, and MCP-1 levels and significantly associated with CVD event history during the 2-years followup period independently of other examined CVD risk factors
(Stepanova et al., 2020c). Taken together, these findings have provided preliminary clinical evidence that elevated POx concentration is associated with increased levels of blood oxidative stress and proinflammatory markers and a high CVD risk in patients with ESRD.

On the other hand, oxidative stress and chronic inflammation, which are present in all CKD stages, with the highest levels in ESRD (Cobo et al., 2018; Vasylchenko et al., 2020), might themselves contribute to the reduced SLC26A6-mediated transcellular oxalate transport and the enhanced passive paracellular intestinal oxalate absorption (Amin et al., 2018; Bashir et al., 2019; Kumar et al., 2021). In an obese mouse model, oxidative stress and systemic/intestinal inflammation were found to reduce active intestinal oxalate secretion and increase passive intestinal oxalate absorption, eventually resulting in obesity-associated hyperoxaluria (Amin et al., 2018; Bashir et al., 2019). Therefore, this vicious circle of complex molecular mechanisms precludes the accurate determination of the root cause of oxalate dyshomeostasis and its relationship with CVD in patients with ESRD. Nevertheless, gut-origin oxidative stress, dyslipidemia, and chronic inflammation remain common links between impaired oxalate homeostasis and increased CVD risk. 


\section{Final Considerations}

In this opinion article, we present a new perspective on the pathophysiologic and translational relevance of impaired oxalate homeostasis in CVD development in patients with ESRD. To the best of our knowledge, although hyperoxalemia and CVD are well-known occurrences in ESRD, few studies have explored this issue. Collectively, we believe that in renal failure conditions, not only do a decrease in the secretion and increase in the reabsorption of oxalate in the kidneys lead to elevated POx but also does the intestine play a crucial role in the oxalate dyshomeostasis. CKD-associated gut dysbiosis with commensal microbiota deficiency leads to decreased total fecal ODA, intestinal barrier dysfunction, and alteration of oxalate transport mechanisms in the intestinal epithelium (both secretion and absorption). Both uremiaand oxalate-induced molecular mechanisms initiated in the gut can be simultaneously involved in the activation of oxidative stress, chronic inflammation, and atherogenesis. However, the role of gut microbiota in oxalate homeostasis

\section{REFERENCES}

Abhishek, A., Benita, S., Kumari, M., Ganesan, D., Paul, E., Sasikumar, P., et al. (2017). Molecular Analysis of Oxalate-Induced Endoplasmic Reticulum Stress Mediated Apoptosis in the Pathogenesis of Kidney Stone Disease. J. Physiol. Biochem. 73, 561-573. doi:10.1007/s13105-017-0587-8

Amin, R., Asplin, J., Jung, D., Bashir, M., Alshaikh, A., Ratakonda, S., et al. (2018). Reduced Active Transcellular Intestinal Oxalate Secretion Contributes to the Pathogenesis of Obesity-Associated Hyperoxaluria. Kidney Int. 93, 1098-1107. doi:10.1016/j.kint.2017.11.011

Arafa, A., Eshak, E. S., and Iso, H. (2020). Oxalates, Urinary Stones and Risk of Cardiovascular Diseases. Med. Hypotheses 137, 109570. doi:10.1016/j.mehy. 2020.109570

Arvans, D., Jung, Y.-C., Antonopoulos, D., Koval, J., Granja, I., Bashir, M., et al. (2017). Oxalobacter Formigenes-Derived Bioactive Factors Stimulate Oxalate Transport by Intestinal Epithelial Cells. Jasn 28, 876-887. doi:10.1681/ASN. 2016020132

Bashir, M., Meddings, J., Alshaikh, A., Jung, D., Le, K., Amin, R., et al. (2019). Enhanced Gastrointestinal Passive Paracellular Permeability Contributes to the Obesity-Associated Hyperoxaluria. Am. J. Physiology-Gastrointestinal Liver Physiol. 316, G1-G14. doi:10.1152/ajpgi.00266.2018 -G14 doi:

Chen, Y.-Y., Chen, D.-Q., Chen, L., Liu, J.-R., Vaziri, N. D., Guo, Y., et al. (2019). Microbiome-metabolome Reveals the Contribution of Gut-Kidney axis on Kidney Disease. J. Transl Med. 17, 5. doi:10.1186/s12967-018-1756-4

Cobo, G., Lindholm, B., and Stenvinkel, P. (2018). Chronic Inflammation in EndStage Renal Disease and Dialysis. Nephrol. Dial. Transplant. 33, iii35-iii40. doi:10.1093/ndt/gfy175

Demikhov, O., Dehtyarova, I., Rud, O., Khotyeev, Y., Kuts, L., Cherkashyna, L., et al. (2020). Arterial Hypertension Prevention as an Actual Medical and Social Problem. Bangladesh J. Med. Sci. 19, 722-729. doi:10.3329/bjms.v19i4. 46632

Devarajan, A. (2018). Cross-talk between Renal Lithogenesis and Atherosclerosis: An Unveiled Link between Kidney Stone Formation and Cardiovascular Diseases. Clin. Sci. 132, 615-626. doi:10.1042/CS20171574

Dominguez-Gutierrez, P. R., Kusmartsev, S., Canales, B. K., and Khan, S. R. (2018). Calcium Oxalate Differentiates Human Monocytes into Inflammatory M1 Macrophages. Front. Immunol. 9, 1863. doi:10.3389/fimmu.2018.01863

Ermer, T., Eckardt, K.-U., Aronson, P. S., and Knauf, F. (2016). Oxalate, Inflammasome, and Progression of Kidney Disease. Curr. Opin. Nephrol. Hypertens. 25, 363-371. doi:10.1097/MNH.0000000000000229

Ermer, T., Kopp, C., Asplin, J. R., Granja, I., Perazella, M. A., Reichel, M., et al. (2017). Impact of Regular or Extended Hemodialysis and Hemodialfiltration on and CVD is still far from being fully understood. Thus, we believe that our hypothesis would be reflected in further experimental and large-scale research to clarify the interaction mechanisms and the role of impaired oxalate homeostasis in CVD risk in patients with ESRD.

\section{AUTHOR CONTRIBUTIONS}

The author confirms being the sole contributor of this work and has approved it for publication.

\section{FUNDING}

This research did not receive any specific grant from funding agencies in the public, commercial, or not-for-profit sectors. The studies reported in this publication were supported by the Institute's research work.
Plasma Oxalate Concentrations in Patients with End-Stage Renal Disease. Kidney Int. Rep. 2, 1050-1058. doi:10.1016/j.ekir.2017.06.002

Fan, X., Kalim, S., Ye, W., Zhao, S., Ma, J., Nigwekar, S. U., et al. (2017). Urinary Stone Disease and Cardiovascular Disease Risk in a Rural Chinese Population. Kidney Int. Rep. 2, 1042-1049. doi:10.1016/j.ekir.2017.06.001

GaǏseniuk, F. Z., Driianskaia, V. E., Drannik, G. N., Rudenko, M., Lavrenchuk, O. V., Stepanova, N. M., et al. (2013). Proinflammatory Cytokines in Patients with Pyelonephritis. Lik Sprava 20 (10), 1445-1448. doi:10.1007/s00467-0051941-6

Gomathi, S., Sasikumar, P., Anbazhagan, K., Sasikumar, S., Kavitha, M., Selvi, M. S., et al. (2014). Screening of Indigenous Oxalate Degrading Lactic Acid Bacteria from Human Faeces and South Indian Fermented Foods: Assessment of Probiotic Potential. Scientific World J. 2014, 1-11. doi:10. $1155 / 2014 / 648059$

Hatch, M., Cornelius, J., Allison, M., Sidhu, H., Peck, A., and Freel, R. W. (2006). Oxalobacter Sp. Reduces Urinary Oxalate Excretion by Promoting Enteric Oxalate Secretion. Kidney Int. 69, 691-698. doi:10.1038/sj.ki.5000162

Hatch, M., and Freel, R. W. (2008). The Roles and Mechanisms of Intestinal Oxalate Transport in Oxalate Homeostasis. Semin. Nephrol. 28, 143-151. doi:10.1016/j.semnephrol.2008.01.007

Hu, X., Ouyang, S., Xie, Y., Gong, Z., and Du, J. (2020). Characterizing the Gut Microbiota in Patients with Chronic Kidney Disease. Postgrad. Med. 132, 495-505. doi:10.1080/00325481.2020.1744335

Huang, Y., Zhang, Y. H., Chi, Z. P., Huang, R., Huang, H., Liu, G., et al. (2020). The Handling of Oxalate in the Body and the Origin of Oxalate in Calcium Oxalate Stones. Urol. Int. 104, 167-176. doi:10.1159/000504417

Jonassen, J. A., Kohjimoto, Y., Scheid, C. R., and Schmidt, M. (2005). Oxalate Toxicity in Renal Cells. Urol. Res. 33 (5), 329-339. doi:10.1007/s00240-0050485-3

Khan, S. R. (2014). Reactive Oxygen Species, Inflammation and Calcium Oxalate Nephrolithiasis. Transl Androl. Urol. 3, 256-276. doi:10.3978/j.issn.2223-4683. 2014.06.04

Khan, S. R. (2004). Crystal-induced Inflammation of the Kidneys: Results from Human Studies, Animal Models, and Tissue-Culture Studies. Clin. Exp. Nephrol. 8, 75-88. doi:10.1007/s10157-004-0292-0

Korol, L., Stepanova, N., Vasylchenko, V., Snisar, L., Lebid, L., and Kolesnyk, M. (2021). Plasma Oxalic Acid as a Trigger for Oxidative Processes in End-Stage Renal Disease Patients. Ukrainian J. Nephrol. Dial. 1 (69), 46-53. doi:10.31450/ ukrjnd.1(69).2021.07

Kumar, P., Patel, M., Oster, R. A., Yarlagadda, V., Ambrosetti, A., Assimos, D. G., et al. (2021). Dietary Oxalate Loading Impacts Monocyte Metabolism and Inflammatory Signaling in Humans. Front. Immunol. 12, 105. doi:10.3389/ fimmu.2021.617508 
Liu, Y., Li, S., Zeng, Z., Wang, J., Xie, L., Li, T., et al. (2014). Kidney Stones and Cardiovascular Risk: A Meta-Analysis of Cohort Studies. Am. J. Kidney Dis. 64, 402-410. doi:10.1053/j.ajkd.2014.03.017

Ma, J., and Li, H. (2018). The Role of Gut Microbiota in Atherosclerosis and Hypertension. Front. Pharmacol. 9, 9. doi:10.3389/fphar.2018.01082

Matey-Hernandez, M. L., Williams, F. M. K., Potter, T., Valdes, A. M., Spector, T. D., and Menni, C. (2018). Genetic and Microbiome Influence on Lipid Metabolism and Dyslipidemia. Physiol. Genomics 50, 117-126. doi:10.1152/ physiolgenomics.00053.2017

Miller, A. W., Choy, D., Penniston, K. L., and Lange, D. (2019). Inhibition of Urinary Stone Disease by a Multi-Species Bacterial Network Ensures Healthy Oxalate Homeostasis. Kidney Int. 96, 180-188. doi:10.1016/j.kint.2019.02.012

Mitchell, T., Kumar, P., Reddy, T., Wood, K. D., Knight, J., Assimos, D. G., et al. (2019). Dietary Oxalate and Kidney Stone Formation. Am. J. Physiology-Renal Physiol. 316, F409-F413. doi:10.1152/ajprenal.00373.2018

Perinpam, M., Enders, F. T., Mara, K. C., Vaughan, L. E., Mehta, R. A., Voskoboev, N., et al. (2017). Plasma Oxalate in Relation to eGFR in Patients with Primary Hyperoxaluria, Enteric Hyperoxaluria and Urinary Stone Disease. Clin. Biochem. 50, 1014-1019. doi:10.1016/j.clinbiochem.2017.07.017

Ren, Z., Fan, Y., Li, A., Shen, Q., Wu, J., Ren, L., et al. (2020). Alterations of the Human Gut Microbiome in Chronic Kidney Disease. Adv. Sci. 7, 2001936. doi:10.1002/advs.202001936

Robijn, S., Hoppe, B., Vervaet, B. A., D’Haese, P. C., and Verhulst, A. (2011). Hyperoxaluria: a Gut-Kidney axis?. Kidney Int. 80, 1146-1158. doi:10.1038/ki. 2011.287

Stepanova, N., Driianska, V., Korol, L., Snisar, L., Lebid, L., and Korol, L. (2020c). Plasma Oxalic Acid and Cardiovascular Risk in End-Stage Renal Disease Patients: A Prospective, Observational Cohort Pilot Study. Korean J. Intern. Med.. doi:10.3904/kjim.2020.561 Available at: https://www.kjim.org/journal/ view.php?number=170524 (Accessed March 15, 2021).

Stepanova, N., Tolstanova, G., Akulenko, I., Korol, L., Savchenko, O., Snisar, L., et al. (2020a). Regulation of Oxalate Homeostasis by OxalateDegrading Activity in Fecal Microbiota in Dialysis Patients. Nephrology Dialysis Transplantation. gfaa142. 35 (3), P0176. doi:10. 1093/ndt/gfaa142.p0176

Stepanova, N., Tolstanova, G., Akulenko, I., Savchenko, O., Lebid, L., Skovorodka, M., et al. (2020b). Oxalate-degrading Activity in Fecal Microbiota Associated with Blood Lipid Profile in Dialysis Patients. Nephrology Dialysis Transplantation. gfaa139. 35 (3), SO011. doi:10.1093/ndt/gfaa139.so011
Stepanova, N., Tolstanova, G., Driyanska, V., and Kolesnyk, M. (2018). Hyperoxaluria-associated Alterations of the Intestinal Colonization Resistance in Patients with Recurrent Pyelonephritis. Nephrol. Dial. Transplant. 33, i52. doi:10.1093/ndt/gfy104.fp008

Stepanova, N., Tolstanova, G., Korol, L., Akulenko, I., Savchenko, O., and Kolesnyk, M. (2021). A Potential Role of Fecal Oxalate-Degrading Activity in Oxalate Homeostasis in End-Stage Renal Disease Patients; a Descriptive Pilot Study. J. Ren. Inj. Prev. Available at: http://www.journalrip.com/Files/Inpress/ jrip-26815.pdf (Accessed March 15, 2021).

Ticinesi, A., Nouvenne, A., Chiussi, G., Castaldo, G., Guerra, A., and Meschi, T. (2020). Calcium Oxalate Nephrolithiasis and Gut Microbiota: Not Just a GutKidney Axis. A Nutritional Perspective. Nutrients 12, 548. doi:10.3390/ nu12020548

Vasylchenko, V. S., Korol, L. V., Kuchmenko, O. B., and Stepanova, N. M. (2020). The Oxidative Status in Patients with Chronic Kidney Disease. Ukrainian Biochem. J. 92, 70-77. doi:10.15407/ubj92.05.070

Whittamore, J. M., and Hatch, M. (2017). The Role of Intestinal Oxalate Transport in Hyperoxaluria and the Formation of Kidney Stones in Animals and Man. Urolithiasis 45, 89-108. doi:10.1007/s00240-016-0952$\mathrm{z}$

Wolf, D., and Ley, K. (2019). Immunity and Inflammation in Atherosclerosis. Circ. Res. 124, 315-327. doi:10.1161/CIRCRESAHA.118.313591

Yang, X., Li, Y., Li, Y., Ren, X., Zhang, X., Hu, D., et al. (2017). Oxidative StressMediated Atherosclerosis: Mechanisms and Therapies. Front. Physiol. 8, 600. doi:10.3389/fphys.2017.00600

Zhou, W., Cheng, Y., Zhu, P., Nasser, M. I., Zhang, X., and Zhao, M. (2020) Implication of Gut Microbiota in Cardiovascular Diseases. Oxidative Med. Cell Longevity 2020, 5394096. doi:10.1155/2020/5394096

Conflict of Interest: The author declares that the research was conducted in the absence of any commercial or financial relationships that could be construed as a potential conflict of interest.

Copyright (c) 2021 Stepanova. This is an open-access article distributed under the terms of the Creative Commons Attribution License (CC BY). The use, distribution or reproduction in other forums is permitted, provided the original author(s) and the copyright owner(s) are credited and that the original publication in this journal is cited, in accordance with accepted academic practice. No use, distribution or reproduction is permitted which does not comply with these terms. 